\title{
Identification of Hub Genes Associated with the Development of Acute Kidney Injury by Weighted Gene Co-Expression Network Analysis
}

\author{
Xiao Lin Jianchun Li Ruizhi Tan Xia Zhong Jieke Yang Li Wang \\ Laboratory of Organ Fibrosis Prophylaxis and Treatment by Combine Traditional Chinese and Western Medicine, \\ Research Center of Integrated Traditional Chinese and Western Medicine, Affiliated Traditional Medicine Hospital, \\ Southwest Medical University, Luzhou, China
}

\section{Keywords}

Weighted gene co-expression network analysis · Acute kidney injury · Rplp1 - Lgals1

\begin{abstract}
Background: Acute kidney injury (AKI) is a severe clinical syndrome, causing a profound medical and socioeconomic burden worldwide. This study aimed to explore underlying molecular targets related to the progression of AKI. Methods: A public database originated from the NCBI GEO database (serial number: GSE121190) and a well-established and unbiased method of weighted gene co-expression network analysis (WGCNA) to identify hub genes and potential pathways were used. Furthermore, the unbiased hub genes were validated in 2 classic models of AKI in a rodent model: chemically established AKI by cisplatin- and ischemia reperfusioninduced AKI. Results: A total of 17 modules were finally obtained by the unbiased method of WGCNA, where the genes in turquoise module displayed strong correlation with the development of AKI. In addition, the results of gene ontology revealed that the genes in turquoise module were involved in renal injury and renal fibrosis. Thus, the hub genes were further validated by experimental methods and primarily obtained Rplp1 and Lgals 1 as key candidate genes
\end{abstract}

karger@karger.com www.karger.com/kbr

Karger $\stackrel{\text { ' }}{5}$

BOPEN ACCESS
(C) 2021 The Author(s)

Published by S. Karger AG, Basel

This article is licensed under the Creative Commons AttributionNonCommercial-NoDerivatives 4.0 International License (CC BY NC-ND) (http://www.karger.com/Services/OpenAccessLicense) Usage and distribution for commercial purposes as well as any distribution of modified material requires written permission. related to the progression of AKI by the advantage of quantitative PCR, Western blotting, and in situ tissue fluorescence. Importantly, the expression of Rplp1 and Lgals 1 at the protein level showed positive correlation with renal function, including serum $\mathrm{Cr}$ and BUN. Conclusions: By the advantage of unbiased bioinformatic method and consequent experimental verification, this study lays the foundation basis for the pathogenesis and therapeutic agent development of AKI.

(c) 2021 The Author(s)

Published by S. Karger AG, Basel

\section{Introduction}

Acute kidney injury (AKI) is a common clinical syndrome, including a variety of pathophysiological processes $[1,2]$. Actually, the recovery of renal function in AKI is often incomplete and gradually develops into CKD, which eventually evolves into ESRD [3]. AKI is a pivotal contributor of CKD and consequent ESRD [4]. In the progression of AKI transitions into CKD, structural abnormalities are manifested as matrix expansion depositing in both mesangium and tubulointerstitium. Targeting

Xiao Lin, Jianchun Li, and Ruizhi Tan contributed equally to this work. 
Table 1. The primers used for qRT-PCR

\begin{tabular}{lll}
\hline Gene name & Primer sequence $\left(5^{\prime} \rightarrow 3^{\prime}\right)$ & \\
\hline Lgals1 & F:AACCTGGGGAATGTCTCAAAGT & R:GGTGATGCACACCTCTGTGA \\
Col3a1 & F:CTGTAACATGGAAACTGGGGAAA & R:CCATAGCTGAACTGAAAACCACC \\
Col6a2 & F:GCTCCTGATTGGGGGACTCT & R:CCAACACGAAATACACGTTGAC \\
Rplp1 & F:CTCGCTTGCATCTACTCCGC & R:AGAAAGGTTCGACGCTGACAC \\
Mgp & F:GGCAACCCTGTGCTACGAAT & R:CCTGGACTCTCTTTTGGGCTTTA \\
\hline
\end{tabular}

on renal fibrosis after AKI has been considered to be a therapeutic approach to minimize the transitions [5-7].

Elevated plasma $\mathrm{Cr}$ is considered to be a classic criterion for diagnosing $\mathrm{AKI}$ in the past decades. But it may not be a proper biomarker since it often suggests the balance of production and excretion of $\mathrm{Cr}$ [8]. Although eliminating the short- and long-term outcomes of AKI is urgent, therapeutic therapies and early detection biomarkers are still limited. In addition, there are presently no clinically valid biomarkers for the diagnosis of renal fibrosis after AKI. Hence, it is beneficial to develop more therapeutic therapies and figure out the potential biomarkers for the treatment of fibrosis and delaying the progression $[1,8]$.

Due to flawed methodologies of molecular biology, there are still certain limitations in the comprehensive exploration of the entire biological system in diseases [9]. As a systematic biological method for describing the genetic association patterns between different samples, weighted gene co-expression network analysis (WGC$\mathrm{NA}$ ) is mainly used to screen and identify highly collaborative modules. According to the internality of the module and the relationship between modules and phenotypes, it has been widely used in the study of omics analysis to mine potential biomarkers or therapeutic targets [10-12]. Compared with data that only focus on differential expression, WGCNA has the following advantages: it can take full advantage of information, associate interesting alternations of phenotypes, and avoid the defects of differential expression analysis artificially setting thresholds. What is more, WGCNA can not only analyze the mRNA level of samples but also be used for microRNA and proteomics research, which is of great help in finding potential biological markers and therapeutic targets $[13,14]$.

In the present study, we performed a well-established bioinformatic method - WGCNA - to analyze the expression profile (NCBI GEO serial number: GSE121190) and aimed to identify the potential pathways and genes associated with the progression of AKI. Importantly, we used experimental methods to further verify the hub genes, so as to provide new insights for the occurrence, development, and treatment of AKI.

\section{Materials and Methods}

\section{Data Preprocessing}

The data set was obtained from the GEO database (https:// www.ncbi.nlm.nih.gov/geo/). In this study, 12 samples of GSE121190 data set were used for WGCNA analysis. Twelve samples included control group and folic acid-induced 3-/7-/14-day AKI group (3 samples per group). The R package "limma" is used for background correction and normalization processing with the original data. If multiple probes match with 1 gene, the average value is calculated as the gene expression value $[9,15]$.

Co-Expression Network Construction and Functional Analysis

Then, the soft threshold is calculated by WGCNA algorithm. The scale-independent value and average connectivity of modules under different soft thresholds were tested by the gradient method in order to determine the appropriate soft threshold $[13,16,17]$. By selecting appropriate soft threshold values, the co-expression network was constructed to ensure the authenticity of the results, and the minimum number of genes in each module was not $<30$. Then, use the R package "Clusterprofiler" toolkit to perform GO analysis [17-19].

\section{Verification of Hub Gene}

Next, we analyzed the hub genes according to the threshold value in each module and visualized using Cytoscape software. The hub genes were treated as the following criterion: (a) absolute value of gene significance (GS), FA 3/7/14 days $\geq 0.2$, and (b) the genes in the main module were analyzed by the STRING database (http:// www.string-db.org/) to generate the protein-protein interaction (PPI) network, and the top 5 genes of highest connectivity were treated as hub genes for further verification [20].

Cisplatin- and Hypoxia-Ischemia-Induced Animal Models

Male C57BL/6 mice (SPF level, 6 8-week-old, 20 25 g) were purchased from Chengdu Dashuo Experimental Animal Co., Ltd. (SCXK [Sichuan] 2015-030) and housed and bred under standard conditions of care at the Experimental Animal Center of Southwest Medical University (SYXK [Sichuan] 2018-065). The mice
Lin/Li/Tan/Zhong/Yang/Wang 
A
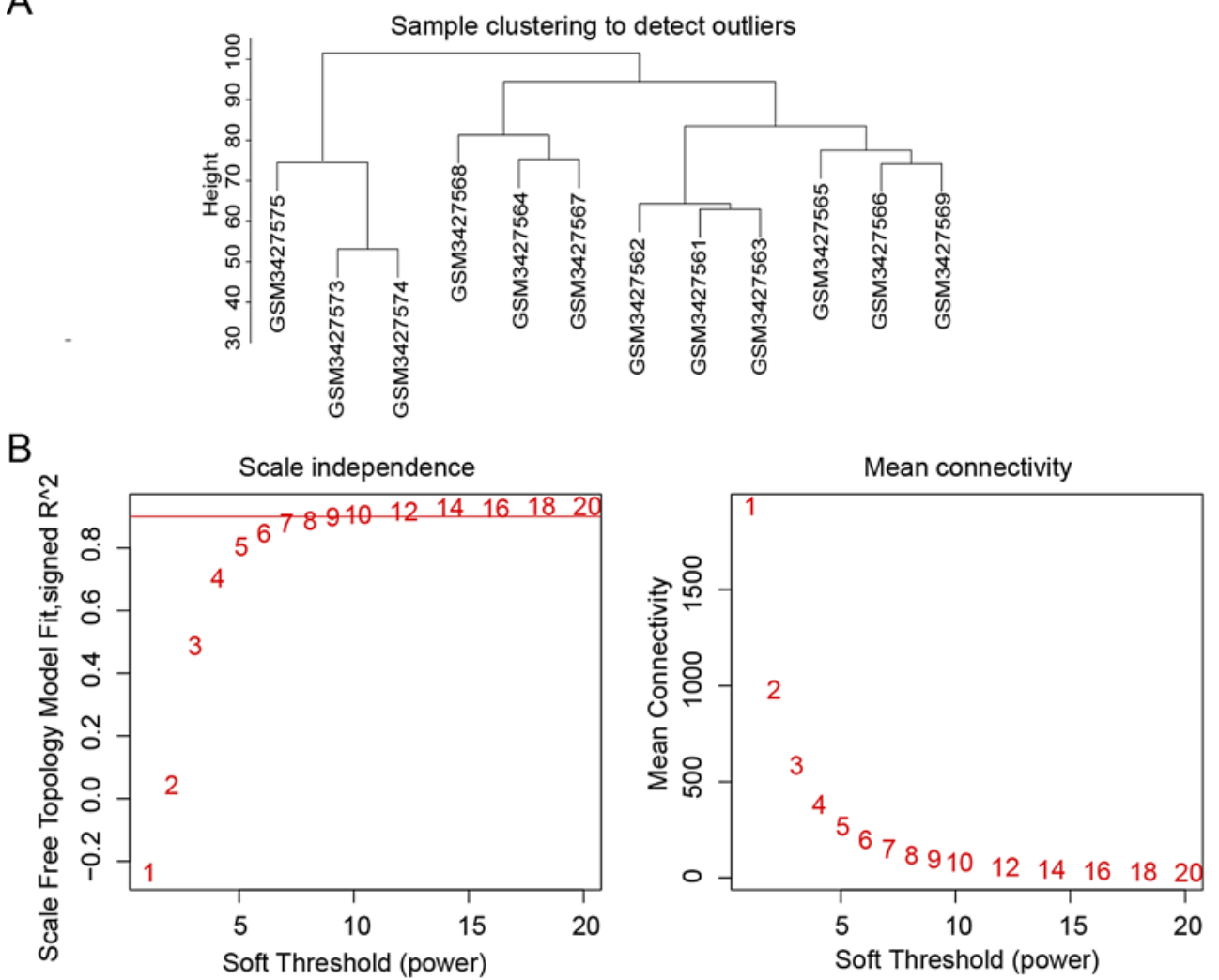

C

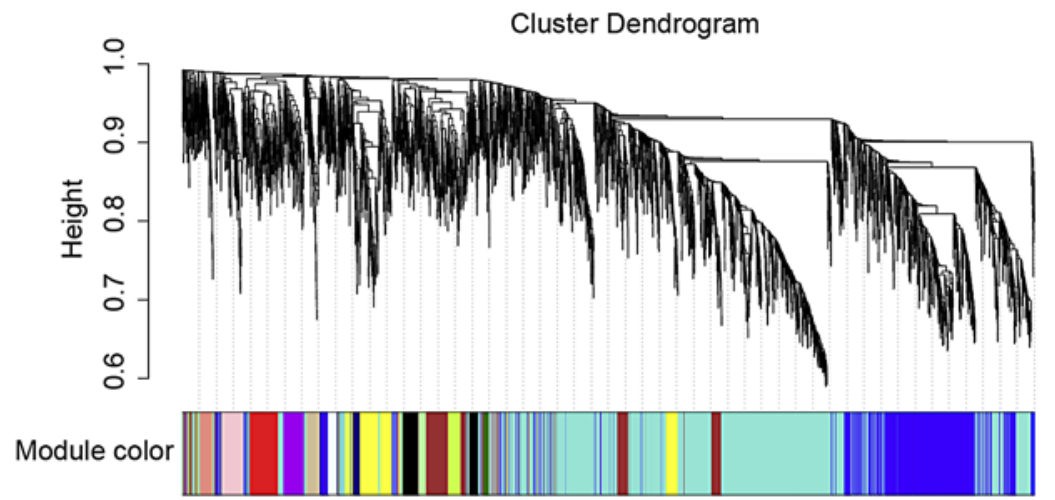

Fig. 1. Construction of collaborative expression module. A Clustering dendrogram of 12 samples. B Analysis of network topology for various soft thresholding (power). C Co-expression module constructed by dynamic hybrid algorithm. Every leaf node represents a gene, and the different colors represent different modules in the tree diagram.

were classified into 3 groups ( $n=6$ per group): control, Cis-AKI, and IRI-AKI; mice in the Cis-AKI group were injected cisplatin (Sigma-Aldrich, USA) $(15 \mathrm{mg} / \mathrm{kg}$ ) by intraperitoneal injection [21]. Mice in the IRI group were anesthetized with $1 \%$ pentobarbital sodium and placed on a small thermostatic electric blanket to keep body temperature at $36^{\circ} \mathrm{C}$. After anesthesia, bilateral flanking incisions were taken, and the arteriovenous arteries were clamped with arterial clips for $35 \mathrm{~min}$. Saline was added dropwise to keep the kidneys moist every $5 \mathrm{~min}$. After the arterial clip was removed, the incisions were sutured, and the mice were placed in $37^{\circ} \mathrm{C}$ environment $[22,23]$. At the end of the experimental period, the mice were killed at days 3 and 7, respectively. Mouse blood samples were 
Table 2. Number of genes in 17 co-expression modules

\begin{tabular}{lr}
\hline Modules & Freq \\
\hline Light cyan & 71 \\
Midnight blue & 75 \\
Cyan & 84 \\
Salmon & 85 \\
Tan & 86 \\
Green yellow & 94 \\
Magenta & 99 \\
Purple & 99 \\
Pink & 106 \\
Red & 130 \\
Black & 130 \\
Green & 206 \\
Yellow & 297 \\
Brown & 421 \\
Gray & 524 \\
Blue & 1,219 \\
Turquoise & 1,620 \\
\hline
\end{tabular}

obtained before the cervical dislocation. All experimental procedures were approved by the Ethics Committee of Southwest Medical University (approval number: 201812-55).

\section{Histological Analysis and Detection of Renal Function}

The kidney was made into paraffin sections according to conventional methods. Briefly, the kidney tissues were fixed with $10 \%$ formaldehyde, dehydrated, and embedded in paraffin [24]. The sections were dehydrated and stained with hematoxylin and eosin (Beyotime, China). Ultimately, the sections were photographed with a virtual slide microscope (VS120-S6-W, Olympus, Japan). At the same time, serum $\mathrm{Cr}$ and BUN were measured according to the kit instructions (Nanjing JianCheng, China).

\section{RNA Isolation and Quantitative PCR}

Total RNA was extracted using an RNA extraction kit (Tiangen, Beijing) and complementary DNA was obtained using the Reverse Transcription Kit (Promega, Shanghai, China) according to the manufacturer's instructions. Then, quantitative PCR was performed in LightCycler ${ }^{\circledR} 480$ II Real-Time PCR System (Roche, Germany). Primers were synthesized by Sangon Biotech (Shanghai, China), and sequences are available in Table 1.

\section{Western Blot}

Total proteins were isolated and dissolved in RIPA buffer. Next, protein concentrations were detected using a BCA Protein Assay Kit (Beyotime Biotechnology, China). The protein samples were run on $12 \%$ sodium dodecyl sulfate-polyacrylamide gel and transferred to PVDF membranes (Millipore, Danvers, MA, USA). Membranes were blocked with $5 \%$ bovine serum albumin at room temperature for $1 \mathrm{~h}$ and incubated at $4^{\circ} \mathrm{C}$ overnight with the indicated primary antibodies: anti-Lgals1 (Cell Signaling Technology, Danvers, MA, USA, 1:1,000) and anti-Rplp1 antibodies (Invitrogen, Waltham, MA, USA, 1:500). Afterward, PVDF membranes were washed with Tris-buffered Saline Tween and incubated at

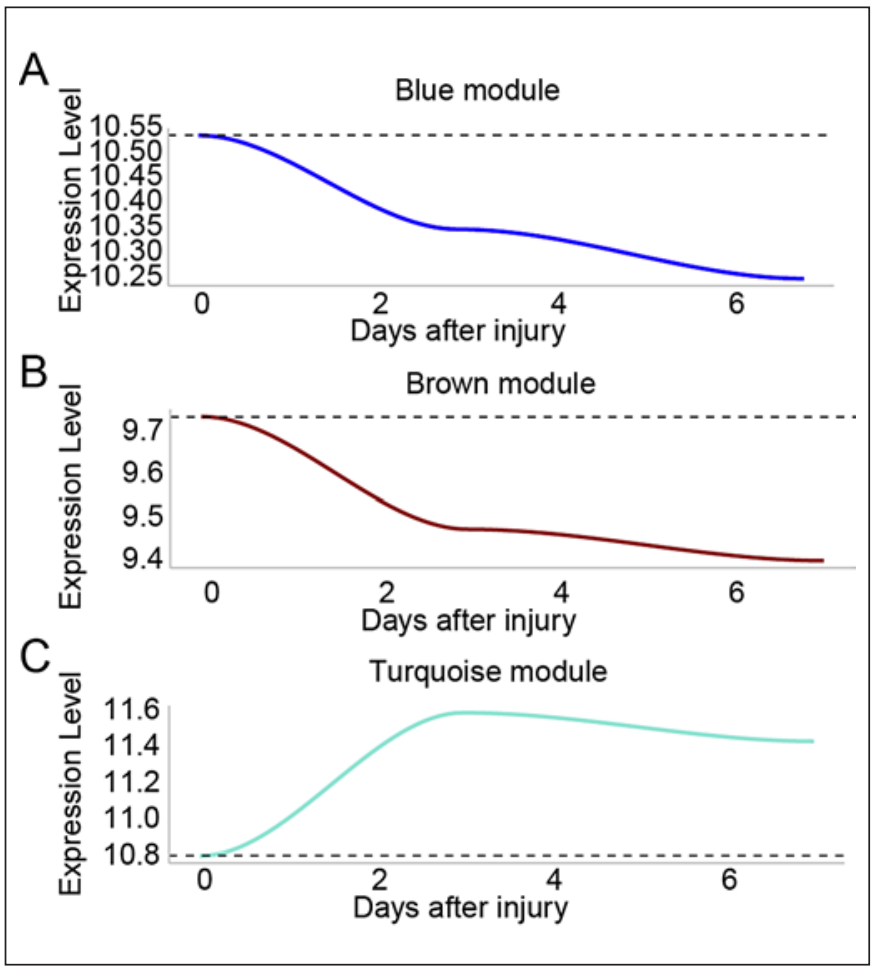

Fig. 2. The fitting curve in 3 main modules. A-C Averaged expression levels of 30 genes within blue, brown, and turquoise modules, respectively, at all time points.

room temperature for $1 \mathrm{~h}$ with a secondary antibody (Invitrogen, Waltham, MA, USA; 1:10,000). Bands were visualized using the ECL Chemiluminescence Kit (Thermo, Waltham, MA, USA) and analyzed using ImageJ software.

\section{In situ Immunofluorescence}

For tissue immunofluorescence, kidney tissues were fixed with $10 \%$ formaldehyde solution and dehydrated, respectively, with 10 , 20 , and $30 \%$ sucrose, followed by embedding in OCT. The samples were then made into $4-\mu \mathrm{m}$ sections and washed 3 times with PBS for $5 \mathrm{~min}$. The tissues were blocked with 5\% BSA for $1 \mathrm{~h}$ at room temperature and then incubated at $4^{\circ} \mathrm{C}$ overnight with the indicated primary antibodies: anti-Lgals1 (Cell Signaling Technology, Danvers, MA, USA; 1:200) and anti-Rplp1 antibodies (Invitrogen, Waltham, MA, USA; 1:100). The next day, tissues were washed with PBS for 5 min 3 times, followed by incubation with Alexa Fluor $^{\circledR}$ 488-conjugated secondary antibodies (Cell Signaling Technology, Danvers, MA, USA; 1:200) and at room temperature for $1 \mathrm{~h}$. The images were captured by a virtual slide microscope (VS120-S6-W, Olympus, Japan).

\section{Statistical Analysis}

Real-time PCR and Western blot statistical analyses were performed by GraphPad Prism 7.0 (GraphPad Software, La Jolla, CA, USA). One-way ANOVA was used if more than 2 comparison groups exist. $p<0.05$ was considered as a statistical difference. 


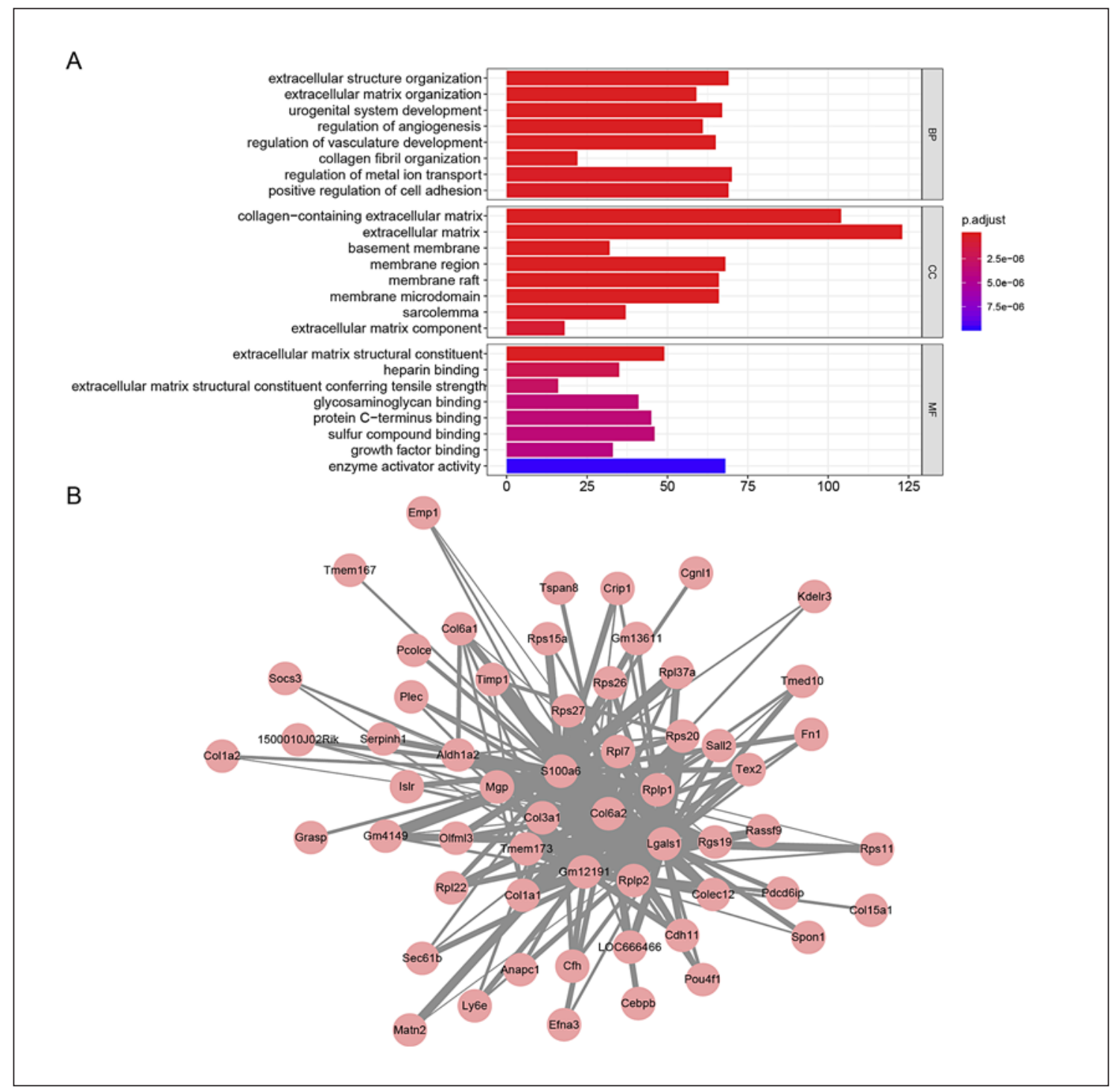

Fig. 3. The functional analysis of the turquoise module. A Gene function enrichment analysis results of the turquoise module. B PPI network of the hub genes in the turquoise module, and the results were visualized by Cytoscape. PPI, protein-protein interaction; ECM, extracellular matrix.

\section{Results}

\section{Data Preprocessing}

The 12 samples of array data in the downloaded data set GSE121190 were preprocessed using the limma package of $\mathrm{R}$ language. In order to obtain genes associated with AKI, it is necessary to compare gene expression changes between normal and AKI samples and screen out the $25 \%$ of the genes before the variance. As a result, a total of 5,418 genes were obtained for the latter WGCNA analysis.

\section{Construction of Collaborative Expression Module}

First of all, samples were first clustered to remove outliers by $\mathrm{R}$ language hclust tool. The results are presented in Figure 1A. Then, we measured the independence and average connectivity of modules at different thresholds. As shown in Figure 1B, it can be judged from the figure that when the threshold value is equal to 8 , which not only makes the average connection degree of the network not too low but also ensures that the network constructed is close to the scale-free network. 
In the next step, we converted the adjacency matrix to a topological overlap matrix to reduce noise and false correlations. A system clustering tree was established through topological overlap matrix, as shown in Figure 1C. And the number of genes in each module was set as no less than 30 by the dynamic hybrid shear algorithm. Finally, 17 modules were obtained from the system cluster tree, and the number of genes contained in each co-expression module is shown in Table 2.

\section{Key Modules and Hub Gene Screening}

Afterward, we analyzed the top 3 modules (brown, blue, and turquoise modules) and sought to find out the hub genes. The expression patterns of genes in the 3 modules were analyzed. As presented in Figure 2, the result revealed that blue and brown modules are negatively correlated with AKI progression, whereas the turquoise module is positively correlated with AKI progression. We then performed the functional analysis in each module and found that the genes contained in the brown, blue, and turquoise modules are strictly related to the kidney, genitourinary system development, some essential metabolic processes, and extracellular matrix (ECM) deposition, which are strictly related to renal fibrosis; in particular, the turquoise module is thought to play an essential role in the development of renal fibrosis after AKI (details presented in Fig. 3A and see online suppl. Fig. 1A, 2A; see www.karger.com/doi/10.1159/000511661 for all online suppl. material). We then sought to find hub genes in each module, and after preliminarily filtering, the top 5 genes - Lgals1, Rplp1, Col3a1, Col6a2, and Mgp - in the turquoise module were treated as hub genes (as shown in Fig. 3B). We also performed a similar analysis in the blue and brown modules but failed to find hub genes considering their expression patterns and GS (as presented in online suppl. Fig. 1B, 2B).

\section{Evaluation of Histological Pathology and Renal Function}

Next, we constructed Cis-AKI and IRI-AKI animal models. As shown in online suppl. Figure 3a, HE staining results suggested that tubular necrosis existed in CisAKI/IRI-AKI models, and the injury accentuated over time. Meanwhile, we could also observe that compared with the control group, a rapid increase in serum $\mathrm{Cr}$ and BUN in a time-dependent manner. Collectively, all these results indicated the successful construction of AKI animal models.

\section{Verification of Hub Gene}

To further confirm the reliability of the WGCNA method and screen hub genes closely related to the progression of renal fibrosis, on the above basis, we aimed to validate the expression of top 5 hub genes in Cis-AKI and IRI-AKI animal models by advantage of real-time PCR, Western blotting, and in situ immunofluorescence. The mRNA expression levels of Lgals1, Rplp1, Col3a1, Col6a2, and Mgp in the kidney of AKI mice were detected by real-time PCR. There are many research reports indicated that Col3a1, Col6a2, and Mgp are related to the process of renal fibrosis $[23,25,26]$. However, for Lgals1 and Rplp1, no studies have been done on renal fibrosis after AKI, so Western blot and in situ immunofluorescence were used to further verify the expression of Lgals1 and Rplp1. As shown in Figure 4B, compared with the normal group, the expression levels of Lgals1 and Rplp1 in CisAKI and IRI-AKI mice were also significantly increased. Furthermore, we evaluated the location of Lgals1 and Rplp1 by in situ immunofluorescence. As shown in Figure 5 , Rplp1 is mainly located in the glomeruli and renal tubular, and Lgals1 distributes in the interstitial and renal tubular. Importantly, we also validated the correlation between Rplp1/Lgals1 and BUN/Cr, and the results indicated that Rplp1/Lgals1 at the protein level were positively correlated with BUN/Cr (all $R>0.5, p<0.05)$ (Fig. 4D-E).

\section{Discussion}

Increasing evidence suggests that AKI will eventually develop into CKD/ESRD, with a higher incidence [4, 27]. So far, there are limited therapies on the transition from AKI to CKD [28-30]. Hence, a better understanding of the pathophysiological mechanism and identification of potential biomarkers are greatly helpful to prevent this transition and improve its prognosis. In this study, we performed WGCNA to analyze microarray data related to the transition from AKI to CKD. A total of 17 modules were obtained, and the main 3 gene modules were employed for further analysis. The results from this study suggested that the 3 gene modules are closely related to

Fig. 4. Validation of the hub genes in the turquoise module. A Quantification of the mRNA expression of Lgals1, Col3a1, Rplp1, Mgp, and Col6a2 by Q-PCR. B Western blot was employed to investigate the indicated protein expression. C Quantification of protein expression by scanning densitometry. ${ }^{* * *} p<0.001 \mathrm{com}-$ pared with the control group. D, E The correlation between Rplp1/ Lgals1 and BUN/Cr (all $R>0.5, p<0.05)$.

(For figure see next page.)
Lin/Li/Tan/Zhong/Yang/Wang 
A
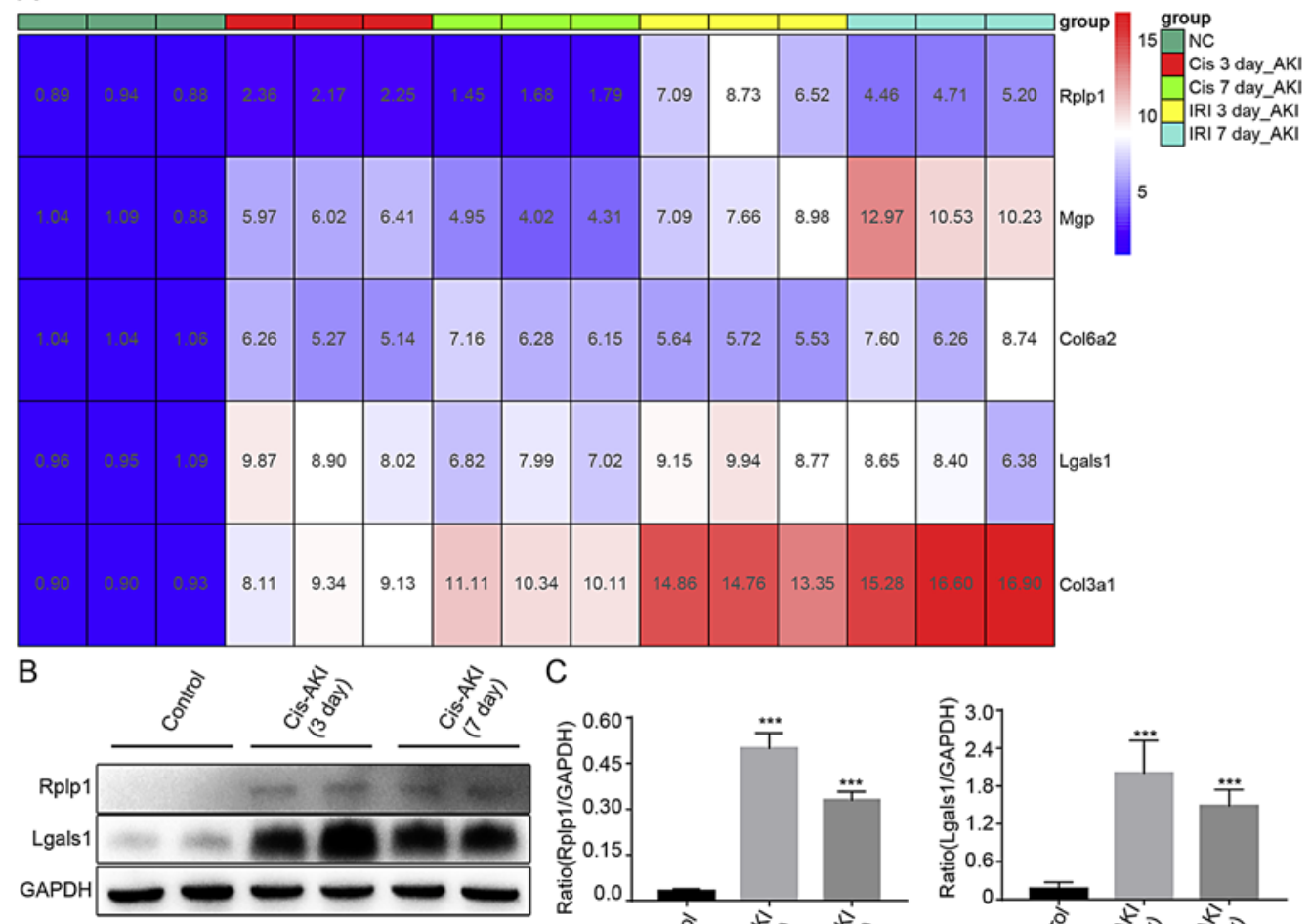

C
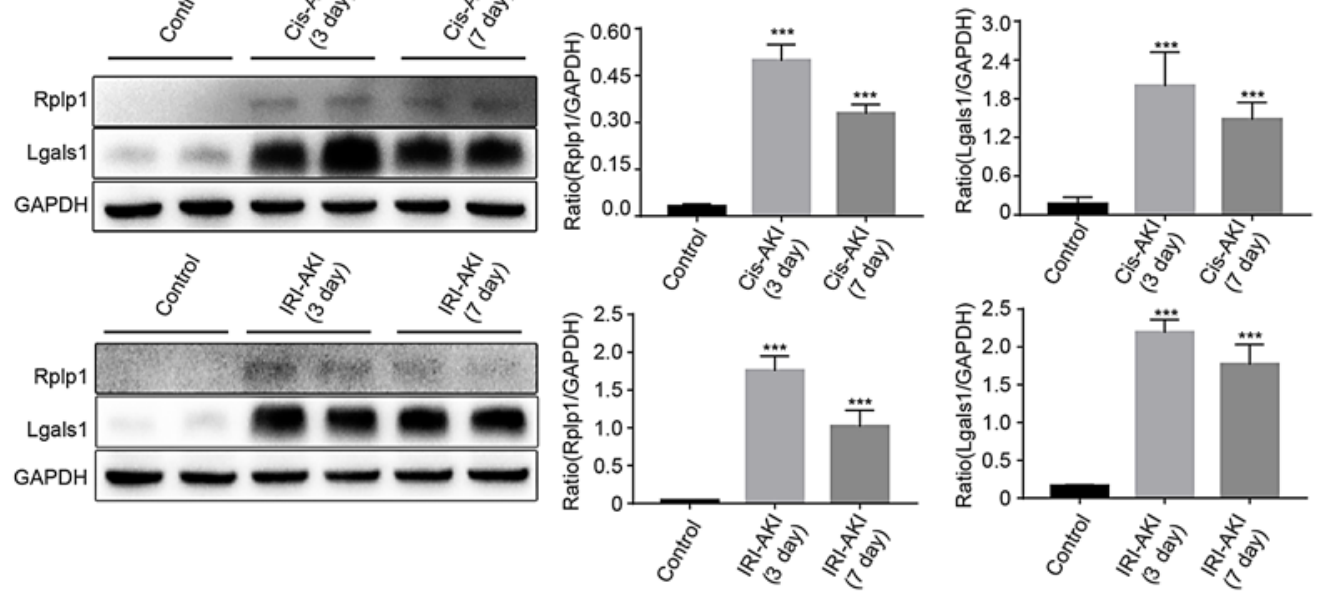

D

$\mathrm{n}=15, \mathrm{r}=0.55$ (person), $\mathrm{p}<0.05$

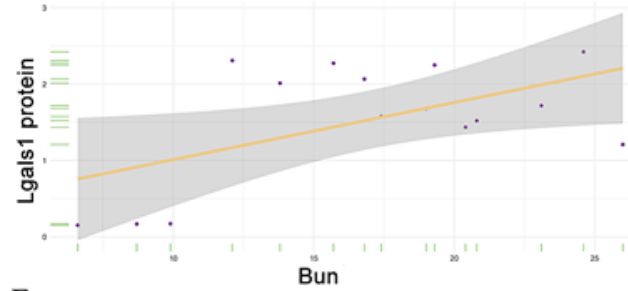

E

$n=15, r=0.74$ (person), $p<0.05$

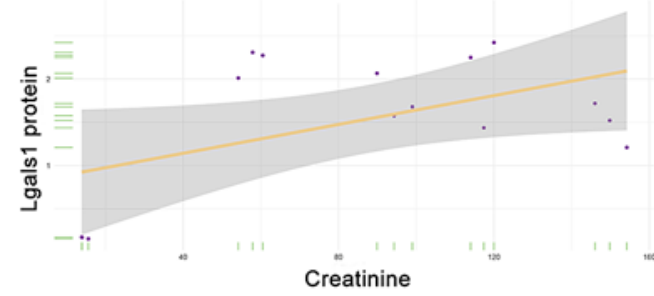

$n=15, r=0.71$ (person), $p<0.05$
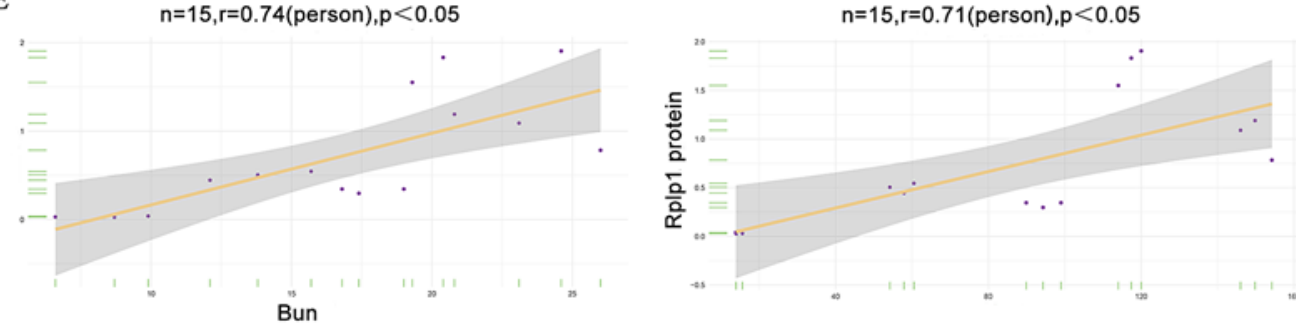

4 


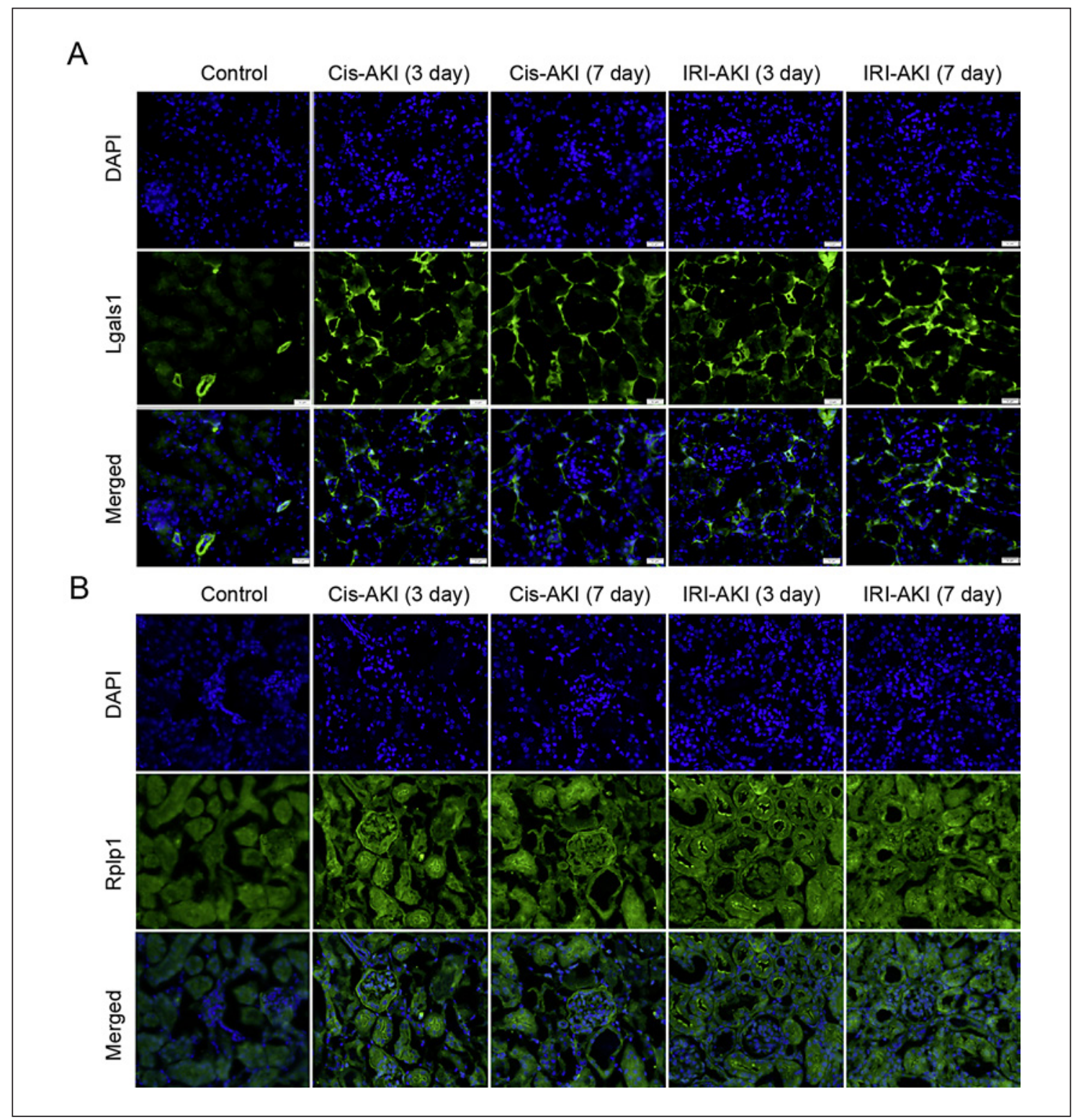

Fig. 5. Validation of the location of the hub genes in the turquoise module. A, B The location and expression of Lgals1 and Rplp1 by in situ immunofluorescence, respectively.

the development of renal fibrosis after AKI in GO classification, for example, renal system development, renal vascular development [31,32], some metabolic processes $[33,34]$, mitochondrial inner membrane, organelle inner membrane, and ECM [35-37]. ECM imbalance is a major cause of renal fibrosis. After AKI, fibrosis is affected by multiple factors such as accumulated inflammatory cells, activated myofibroblasts, and so on $[30,33]$. Therefore, the present study focused on the turquoise module as the module is tightly related to the ECM.
Next, we filtered the genes in the turquoise module by calculating the GS, followed by generating the PPI network. From the PPI network in the turquoise module, it can be concluded that 6 genes S100a6, Col3a1, Col6a2, Mgp, Rplp1, and Lgals1 displayed the highest connectivity with other neighboring genes and located in the center of the PPI network. S100a6 is encoded by being a member of the S100 protein family. It has been reported in the literature that S100a6 may regulate cell proliferation and participate in immune-mediated pathological processes 
of renal epithelial-mesenchymal transition and fibrosis $[38,39]$. Col3a1 and Col6a 2 are collagen type III alpha-1 and collagen type VI alpha-2 chains, respectively. They encode collagen, bind to extracellular machinery proteins, and are extremely important in cell-matrix components. When fibrosis occurs in organs such as liver and kidney, the expression of these collagens is elevated, which is an essential indicator for judging the progress of the disease and the observation of the therapeutic effect $[23,25]$. Mgp, a matrix of Gla protein, is a member of the osteocalcin/Mgp family that is expressed after CKD and is associated with renal prognosis $[26,40]$. In line with previous studies, our research also revealed that S100a6, Col3a1, Col6a2, and Mgp are potential early diagnostic markers of renal fibrosis after AKI.

Rplp1, a gene encoding a ribosomal protein that is part of the $60 \mathrm{~S}$ subunit, plays an important role in the elongation step of protein synthesis. Rplp1 is highly expressed in multiple human cancer cells and its lacking will affect protein synthesis, leading to apoptosis [41, 42]. Lgals1, also known as Galectin-1, is a member of the $\beta$-galactoside-binding protein family and plays a role in regulating apoptosis, cell proliferation, and cell differentiation. Lgals1 is a research hot spot in the field of tumors, and many recent studies have reported that it also plays a vital role in the process of organ fibrosis. It has been indicated that Lgals1 is highly expressed in kidneys of type I and type II diabetes and is a new marker of renal fibrosis [4345]. However, it is worth noting that Rplp1 and Lgals1 have not been reported in renal fibrosis after AKI. In the present study, we have preliminary proved that Rplp1 and Lgals1 might be used as potential biomarkers in renal fibrosis after AKI by bioinformatics and experimental manner. Our multiple experimental analyses indicated that Lgals1 and Rplp1 display dramatically increased in the kidneys of IRI-AKI and Cis-AKI, and the expression of the 2 genes is increased after 3 days and decreased after 7 days, which is consistent with the results analyzed by WGCNA bioinformatics method.

It should be noted that there are several limitations of the study: first, the number of samples used for analysis is relatively insufficient which may omit or decrease the accuracy of analysis. Second, the hub genes were further investigated only in the AKI rodent model rather than human specimens which may also hamper the translation of the findings from the mouse to human. There are many studies on LGALS1 in human cancer, and its expression is elevated in kidney cancer. Targeting LGALS1 can inhibit the development of its tumors. Although RPLP1 has been found in endometriosis, gynecological tumors, and other diseases, it has not been studied in human kidneys. Further investigations of hub genes on human specimens such as urinary excretion may better elucidate their biological role clinically. In conclusion, the present study laid the foundation for further studying the role of Rplp1 and Lgals1 in AKI.

\section{Conclusions}

In summary, this study identified key genes related to AKI through WGCNA analysis, and experimentally found 2 hub genes with potential for further research, Rplp1 and Lgals1. However, the specific mechanism in the pathogenesis of AKI remains further research. The selected critical candidate genes provide new research directions for the prevention, diagnosis, treatment, and prognosis of renal fibrosis.

\section{Statement of Ethics}

All animals were treated in accordance with the National Institutes of Health Guidelines for use and care of research animals.

\section{Conflict of Interest Statement}

The authors declared that there are no conflicts of interest.

\section{Funding Sources}

This work was supported by Luzhou - Southwest Medical Joint Platform Project (2017LZXNYD-P01 and 2018LZXNYD-PT03), the Construction Project of Sichuan Provincial Key Laboratory of Medicine (2018\#53), and the Sichuan Science and Technology Project (2020YJ0442); the Luzhou Municipal - Southwest Medical University Joint Special Grant for the Introduction of High-level Talents (Lan Hui-Yao Team); and the Southwest Medical University and Affiliated Traditional Medicine Hospital Joint Program (2018XYLH-029).

\section{Author Contributions}

Li Wang and Jianchun Li conceived and coordinated the study. Xiao Lin wrote the paper. Jianchun Li revised the paper. Jianchun Li carried out the bioinformatics analysis. Xiao Lin performed the animal experiment and carried out the real-time PCR, IHC, and IF. Xia Zhong and Jieke Yang carried out the WB and collected the mouse renal samples. Ruizhi Tan performed statistical analysis. All authors reviewed the results and approved the final version of the manuscript. 


\section{References}

1 Venkatachalam MA, Weinberg JM, Kriz W, Bidani AK. Failed tubule recovery, AKI$\mathrm{CKD}$ transition, and kidney disease progression. J Am Soc Nephrol. 2015 Aug;26(8): 1765-76.

2 Dong Y, Zhang Q, Wen J, Chen T, He L, Wang $\mathrm{Y}$, et al. Ischemic duration and frequency determines AKI-to-CKD progression monitored by dynamic changes of tubular biomarkers in IRI mice. Front Physiol. 2019; 10:153.

3 Peng Y, Liu L, Wang Y, Yao J, Jin F, Tao T, et al. Treatment with toll-like receptor 2 inhibitor ortho-vanillin alleviates lipopolysaccharide-induced acute kidney injury in mice. Exp Ther Med. 2019 Dec;18(6):482937.

4 Levey AS, James MT. Acute kidney injury. Ann Intern Med. 2017 Nov 7; 167(9):ITC66ITC80.

5 Song Y, Tao Q, Yu L, Li L, Bai T, Song X, et al. Activation of autophagy contributes to the renoprotective effect of postconditioning on acute kidney injury and renal fibrosis. Biochem Biophys Res Commun. 2018; 504(4):641-6.

6 Jaworska K, Ratajczak J, Huang L, Whalen $\mathrm{K}$, Yang M, Stevens BK, et al. Both PD-1 ligands protect the kidney from ischemia reperfusion injury. J Immunol. 2015 Jan 1; 194(1):325-33.

7 Lu M, Wang P, Qiao Y, Jiang C, Ge Y, Flickinger B, et al. GSK3 $\beta$-mediated Keap1-independent regulation of Nrf2 antioxidant response: a molecular rheostat of acute kidney injury to Activation of autophagy contributes to the renoprotective effect of postconditioning on acute kidney injury and renal fibrosis. chronic kidney disease transition. Redox Biol. 2019;26:101275.

8 Chen Y, Lin L, Tao X, Song Y, Cui J, Wan J. The role of podocyte damage in the etiology of ischemia-reperfusion acute kidney injury and post-injury fibrosis. BMC Nephrol. 20193 28;20(1):106.

9 Zuo Z, Shen JX, Pan Y, Pu J, Li YG, Shao XH, et al. Weighted gene correlation network analysis (WGCNA) detected loss of MAGI2 promotes chronic kidney disease (CKD) by podocyte damage. Cell Physiol Biochem. 2018;51(1):244-61.

10 Qin S, Long X, Zhao Q, Zhao W. Co-expression network analysis identified genes associated with cancer stem cell characteristics in lung squamous cell carcinoma. Cancer Invest. 2020 Jan;38(1):13-22.

11 Liang JW, Fang ZY, Huang Y, Liuyang ZY, Zhang XL, Wang JL, et al. Application of weighted gene co-expression network analysis to explore the key genes in Alzheimer's disease. J Alzheimers Dis. 2018;65(4):135364.

12 Kwon J, Kim YJ, Choi K, Seol S, Kang HJ. Identification of stress resilience module by weighted gene co-expression network anal- ysis in Fkbp5-deficient mice. Mol Brain. 201911 27;12(1):99.

13 Yin X, Wang J, Zhang J. Identification of biomarkers of chromophobe renal cell carcinoma by weighted gene co-expression network analysis. Cancer Cell Int. 2018;18:206.

14 Shang J, Wang S, Jiang Y, Duan Y, Cheng G, Liu D, et al. Identification of key lncRNAs contributing to diabetic nephropathy by gene co-expression network analysis. Sci Rep. 2019 Mar 1;9(1):3328.

15 Tang J, Kong D, Cui Q, Wang K, Zhang D, Gong Y, et al. Prognostic genes of breast cancer identified by gene co-expression network analysis. Front Oncol. 2018;8:374.

16 Zhang X, Zhuang J, Liu L, He Z, Liu C, Ma $\mathrm{X}$, et al. Integrative transcriptome data mining for identification of core lncRNAs in breast cancer. PeerJ. 2019;7:e7821.

17 Liu H, Chen P, Jiang C, Han J, Zhao B, Ma $\mathrm{Y}$, et al. Screening for the key lncRNA targets associated with metastasis of renal clear cell carcinoma. Medicine. 2016 Jan;95(2): e2507.

18 Guo Y, Ma J, Xiao L, Fang J, Li G, Zhang L, et al. Identification of key pathways and genes in different types of chronic kidney disease based on WGCNA. Mol Med Rep. 2019 Sep;20(3):2245-57.

19 Pei G, Chen L, Zhang W. WGCNA application to proteomic and metabolomic data analysis. Meth Enzymol. 2017;585:135-58.

20 Chou WC, Cheng AL, Brotto M, Chuang CY. Visual gene-network analysis reveals the cancer gene co-expression in human endometrial cancer. BMC Genomics. 2014 Apr 23;15:300.

21 Tan RZ, Wang C, Deng C, Zhong X, Yan Y, Luo $Y$, et al. Quercetin protects against cisplatin-induced acute kidney injury by inhibiting Mincle/Syk/NF- $\mathrm{kB}$ signaling maintained macrophage inflammation. Phytother Res. 2020 Jan;34(1):139-52.

22 Shih JM, Shih YM, Hou YC, Pai MH, Yeh CL, Yeh SL. Effects of fish oil-based lipid emulsion on inflammation and kidney injury in mice subjected to unilateral hind limb ischemia/reperfusion. Cytokine. 2018 11;111:49-57.

23 Zhou T, Luo M, Cai W, Zhou S, Feng D, Xu $\mathrm{C}$, et al. Runt-related transcription factor 1 (RUNX1) promotes TGF- $\beta$-induced renal tubular epithelial-to-mesenchymal transition (EMT) and renal fibrosis through the PI3K subunit p1108. EBioMedicine. 2018 May;31:217-25.

24 Qin C, Bai Y, Zeng Z, Wang L, Luo Z, Wang $S$, et al. The cutting and floating method for paraffin-embedded tissue for sectioning. J Vis Exp. 2018 Sep 05(139):58288.

25 Guo J, Guan Q, Liu X, Wang H, Gleave ME, Nguan CY, et al. Relationship of clusterin with renal inflammation and fibrosis after the recovery phase of ischemia-reperfusion injury. BMC Nephrol. 2016 Sep 17(171):133.
26 Miyata KN, Nast CC, Dai T, Dukkipati R, LaPage JA, Troost JP, et al. Renal matrix Gla protein expression increases progressively with CKD and predicts renal outcome. Exp Mol Pathol. 2018 08;105(1):120-9.

27 Zuk A, Bonventre JV. Acute kidney injury. Annu Rev Med. 2016;67:293-307.

28 Mehrotra P, Collett JA, McKinney SD, Stevens J, Ivancic CM, Basile DP. IL-17 mediates neutrophil infiltration and renal fibrosis following recovery from ischemia reperfusion: compensatory role of natural killer cells in athymic rats. Am J Physiol Renal Physiol. 2017 Mar 1;312(3):F385-97.

29 Fan Y, Xiao W, Lee K, Salem F, Wen J, He L, et al. Inhibition of reticulon-1A-mediated endoplasmic reticulum stress in early AKI attenuates renal fibrosis development. J Am Soc Nephrol. 2017 Jul;28(7):2007-21.

30 Geng XQ, Ma A, He JZ, Wang L, Jia YL, Shao GY, et al. Ganoderic acid hinders renal fibrosis via suppressing the TGF- $\beta / \mathrm{Smad}$ and MAPK signaling pathways. Acta Pharmacol Sin. 2020 May;41(5):670-7.

31 Verma SK, Molitoris BA. Renal endothelial injury and microvascular dysfunction in acute kidney injury. Semin Nephrol. 2015 Jan;35(1):96-107.

32 Liu S, Soong Y, Seshan SV, Szeto HH. Novel cardiolipin therapeutic protects endothelial mitochondria during renal ischemia and mitigates microvascular rarefaction, inflammation, and fibrosis. Am J Physiol Renal Physiol. 2014 May 01;306(9):F970-80.

33 Sun YB, Qu X, Caruana G, Li J. The origin of renal fibroblasts/myofibroblasts and the signals that trigger fibrosis. Differentiation. 2016 Sep;92(3):102-7.

34 Gong X, Yang C, Hong Y, ACK C, Cai Z. PFOA and PFOS promote diabetic renal injury in vitro by impairing the metabolisms of amino acids and purines. Sci Total Environ. 2019 Aug 1;676:72-86

35 Nguyen-Thanh T, Kim D, Lee S, Kim W, Park SK, Kang KP. Inhibition of histone deacetylase 1 ameliorates renal tubulointerstitial fibrosis via modulation of inflammation and extracellular matrix gene transcription in mice. Int J Mol Med. 2018 Jan;41(1): 95-106.

36 Raup-Konsavage WM, Wang Y, Wang WW, Feliers D, Ruan H, Reeves WB. Neutrophil peptidyl arginine deiminase- 4 has a pivotal role in ischemia/reperfusion-induced acute kidney injury. Kidney Int. 2018 02;93(2): 365-74.

37 Qin J, Peng ZZ, Li Q, Wen R, Tao LJ. Renal fibrosis and mitochondrial damage. Chin Med J. 2018 Nov 20;131(22):2769-72.

38 Li Y, Wagner ER, Yan Z, Wang Z, Luther G, Jiang $\mathrm{W}$, et al. The calcium-binding protein S100A6 accelerates human osteosarcoma growth by promoting cell proliferation and inhibiting osteogenic differentiation. Cell Physiol Biochem. 2015;37(6):2375-92. 
39 Feighery R, Maguire P, Ryan MP, McMorrow T. A proteomic approach to immunemediated epithelial-mesenchymal transition. Proteomics Clin Appl. 2008 Jul;2(7-8): 1110-7.

40 Wei X, Wu W, Li L, Lin J, Liu Q, Gan L, et al. Bone morphogenetic proteins $2 / 4$ are upregulated during the early development of vascular calcification in chronic kidney disease. Biomed Res Int. 2018;2018:8371604.
41 Artero-Castro A, Castellvi J, García A, Hernández J, Ramón y Cajal S, Lleonart ME. Expression of the ribosomal proteins Rplp0, Rplp1, and Rplp2 in gynecologic tumors. Hum Pathol. 2011 Feb;42(2):194-203.

$42 \mathrm{He}$ Z, Xu Q, Wang X, Wang J, Mu X, Cai Y, et al. RPLP1 promotes tumor metastasis and is associated with a poor prognosis in triplenegative breast cancer patients. Cancer Cell Int. 2018;18:170.

43 Al-Obaidi N, Mohan S, Liang S, Zhao Z, Nayak BK, Li B, et al. Galectin-1 is a new fibrosis protein in type 1 and type 2 diabetes. FASEB J. 2019 Jan;33(1):373-87.
44 Arda-Pirincci P, Sacan O, Ozal-Coskun C, Aykol-Celik G, Karabulut-Bulan O, Yanardag R, et al. Galectin-1 exhibits a protective effect against hepatotoxicity induced by dextran sulfate sodium in mice. Hum Exp Toxicol. 2020 Apr;39(4):423-32.

45 Liu Y, Long L, Yuan F, Liu F, Liu H, Peng Y, et al. High glucose-induced Galectin-1 in human podocytes implicates the involvement of Galectin-1 in diabetic nephropathy. Cell Biol Int. 2015 Feb;39(2):217-23. 